\title{
The Analysis of Human Sweat Proteins by Isoelectric Focusing. I. Sweat Collection Utilizing the Macroduct System Demonstrates the Presence of Previously Unrecognized Sex-Related Proteins
}

\author{
DONALD A. SENS. MARGARET A. SIMMONS. AND SAMUEL S. SPICER \\ Department of Pathology; Medical University of South Carolina. Charleston. South Carolina 29425
}

\begin{abstract}
The presence of high concentrations of sodium in the sweat of patients with cystic fibrosis is widely accepted by all investigators as one of the most important manifestations of the genetic defect. However, the simple means of sweat collection utilized for diagnostic purposes has been extended to the biochemical and physical analysis of the macromolecular components of human sweat. The most popular technique for the attainment of sweat has been collection of sweat droplets from the skin surface following heat stimulation. In this report, it is demonstrated that a far superior means of sweat collection exists based on a new collection device called the MACRODUCT. Utilizing this device, in conjunction with thin-layer isoelectric focusing, demonstrated that human sweat contains sexspecific proteins expressed in adult males and absent or greatly decreased from females and prepubertal males. Additionaly, results are presented which suggest that the presence of epidermal keratins are an excellent marker for sweat contaminated with nonsweat gland-derived macromolecules. (Pediatr Res 19: 873-878, 1985)
\end{abstract}

Abbreviations

$\mathrm{CF}$, cystic fibrosis

IEF, isoelectric focusing

The presence of high concentrations of sodium in the sweat of patients with $C F$ is widely accepted by all investigators as one of the most important manifestations of the genetic defect $(1,2)$. This has understandably led to the biochemical and physical analysis of the fluid produced by these structures in hopes of further clucidating the primary abnormality in cystic fibrosis. These studies have ranged from the electrophoretic analysis of the proteins present in human sweat $(3-5)$, to the examination of swcat gland function in vivo $(6,7)$; including the possible production of CF factors by sweat glands and the effect of this CF sweat on the function of isolated, normal sweat glands (8). While the micropuncture studies of Schulz (6) clearly demonstrated the sweat gland to be the source of altered sweat electrolytes in CF patients; further studies analyzing sweat proteins and

Received December 26. 1984: accepted April 2.1985.

Reprint requests Donald A. Sens. Department of Pathology. Medical University of South Carolina. 17I Ashley Avenue. Charleston. SC 29425.

Supported by PHS Grant AM 11028. the possible presence of $\mathrm{CF}$ factors have all failed to assure that the sweat obtained was indeed from the eccrine sweat gland. The very question of the purity of the sweat obtained for some of the above studies is so basic as to require the definition of what is meant by the word sweat.

Human skin contains an abundance of eccrine sweat glands that are found in nearly all exposed skin areas and function to dissipate body heat through evaporation. In addition to these sweat glands are the sebaceous glands. closely associated with hair follicles, and present overall in slightly fewer numbers than the sweat glands. These glands produce an oily secretion. which reaches the skin surface, called sebum which is thought to arise from the disintegration of the alveolar cells of the gland. Depending on the site of sweat collection (such as the forehead) sebaceous glands may outnumber sweat glands or in other sites (such as the forearm) sweat glands may greatly outnumber sebaceous glands. Thus, sweat collected from simple dripping from the skin surface can logically be expected to contain macromolecular substances from both the eccrine sweat glands and the sebaceous glands. In addition, the very nature of human skin further complicates the collection of sweat uncontaminated with other macromolecular components. As skin matures, it undergoes terminal differentiation to form an outer protective layer called the stratum corneum. This surface layer is composed of dead, shedding cells known to contain abundant amounts of keratin and possibly other components which would logically be thought to contaminate sweat samples obtained by simple collection from a wide area of skin surface. Thus, for the purpose of this report, methodology was tested which would appear to yield sweat with a high probability of originating only from the eccrine sweat gland; in line with a definition that sweat is in fact a product of the sweat glands and not other glandular and skinderived components. In this report, it will be demonstrated by IEF profiles that sweat can be collected free of contaminating substances, that heat-induced and pilocarpine-induced sweat are very similar in protein composition, that sex-related differences exist, that serum proteins are reduced in concentration in carefully collected sweat and that the presence of keratin is a superior marker for sweat collected under suboptimum conditions.

\section{MATERIALS AND METHODS}

Sweat Collection. The sweat samples utilized in this study were obtained from normal healthy volunteers and collection was approved by the Internal Review Board for Human Research of the Medical University of South Carolina. Human sweat was 
collected utilizing two different methods. The first method utilized the MACRODUCT (Wescor Co., Logan, UT) sweat collection system with the collection device attached to the upper forearm area. The MACRODUCT consists of a round, $2.8 \mathrm{~cm}$ diameter, plastic concave base with a central aperture through which sweat collects into coiled capillary tubing. The capillary tubing containing the sweat sample is removed, sealed, and transported to the laboratory without risk of contamination or evaporation. Both pilocarpine-induced and heat-induced (heavy exercise or sauna bath) sweat were collected in this manner and will be referred to as pure sweat. Alternatively, heat-induced sweat was obtained directly from the skin surface (the forehead and forearm) using a freshly cleaned and rinsed glass vial. These samples were centrifuged at $8000 \times g$ for 5 min to remove cellular debris. These samples will be referred to as crude sweat. In both cases, the skin area utilized for sweat collection was washed with soap and water, rinsed with $95 \%$ ethanol, and exhaustively rinsed with distilled water. For selected experiments, sweat samples were concentrated by the method of Saul and Don (9).

IEF. IEF was performed utilizing an LKB Multiphore System consisting of an electrophoresis apparatus (model 2117, LKB, Bromma, Sweden), a power supply unit (model 2103, LKB), and a circulatory cooling apparatus (Multi-Temp 2209, LKB). IEF was performed at constant power utilizing $5 \%$ acrylamide gel slabs containing $3.5 \%$ monomer concentration of methylene bisacrylamide with ampholine mixtures (LKB) chosen to represent various $\mathrm{pH}$ ranges between 2.5 to 11.0 . The slab gels were prepared on $125 \times 130 \times 1 \mathrm{~mm}$ glass plates (LKB no. 2117-104) which had been pretreated with $0.1 \%$ silane (Pharmacia, Piscataway, NJ) in acetone (Fisher Scientific, Norcross, GA) for 5 min. The resulting slab gels were $0.50 \mathrm{~mm}$ thick, $9.5 \mathrm{~mm}$ in length, and $115 \mathrm{~mm}$ wide. The cathodal wick buffer was $1.0 \mathrm{M}$ $\mathrm{NaOH}$ (Sigma Chemical Co., St. Louis, MO) and the anodal wick buffer was $1.0 \mathrm{M} \mathrm{H}_{3} \mathrm{PO}_{4}$ (Fisher Scientific). The sweat samples were applied on $5 \times 10 \mathrm{~mm}$ rectangular sample application pieces (LKB, no. 1850-901) positioned $1.0 \mathrm{~cm}$ below the cathodal wick. The sample volume applied was $20 \mu$ l. The gel was focused for $2 \mathrm{~h}$ at $5 \mathrm{~W}$ with a final voltage of $1500 \mathrm{~V}$. The sample application pieces were removed after $1 \mathrm{~h}$ of electrofocusing.

Following IEF, the gels were silver-stained employing the method of Merrill et al. (10) as modified by Emerson et al. (11). Following silver staining, the gels were scanned utilizing an UltroScan Laser Densitometer (LKB).

Immuno-dot. The human sweat samples were tested by the Immuno-Blot (Bio-Rad, Richmond, CA) technique (12) for the presence of human serum proteins and keratin as described by the manufacturer. Rabbit antiserum against human serum proteins and human keratin were obtained from Accurate Chemical and Scientific Corporation (San Diego, CA). Briefly, 4 to $6 \mu \mathrm{l}$ of sweat sample, not subjected to IEF, was "dotted" onto a nitrocellulose filter $1 \mu \mathrm{l}$ at a time with drying allowed to occur between each addition. The membrane was then immersed at a $45^{\circ}$ angle

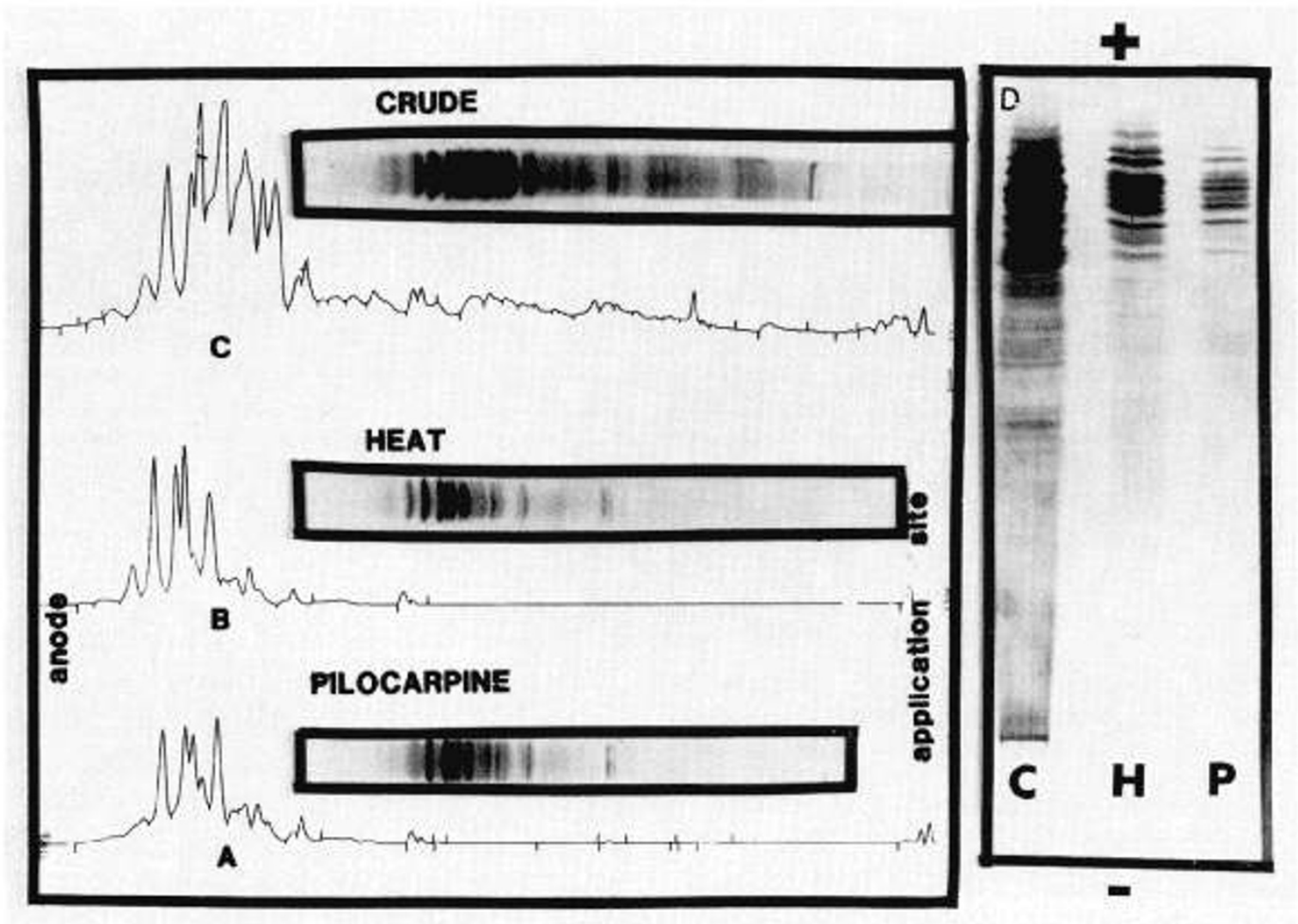

Fig. 1. A comparison of the electrofocusing profile of adult sweat collected utilizing the MACRODUCT system versus droplet collection. The IEF profile of both adult male and female sweat collected by dripping from the forearm during exercise was compared to that obtained using the MACRODUCT collector following both exercise and pilocarpine stimulation. For each sample $20 \mu \mathrm{l}$ of sweat was utilized for IEF over a pH range of 3.5 to 9.0. Following completion of the IEF run, the resulting gel was silver stained for visualization of proteins. $A$, the IEF profile and resulting scan of an adult male sweat sample obtained utilizing the MACRODUCT collection system following pilocarpine stimulation. The site of collection was the forearm. $B$, identical to $A$ except exercise was utilized in place of pilocarpine to stimulate sweating. $C$, identical to $B$ except sweat was obtained, following exercise, by droplet collection from the forearm skin surface. $D$, identical to $A$-C except adult female sweat samples were utilized and gel scans were not depicted. $C$, crude sweat obtained by droplet collection during exercise; $H$, sweat obtained utilizing the MACRODUCT collector during exercise; $P$, sweat obtained utilizing the MACRODUCT collector following pilocarpine stimulation. The results shown were representative of all 13 male and 11 female sweat samples analyzed and all were analyzed in triplicate with consistent results. 


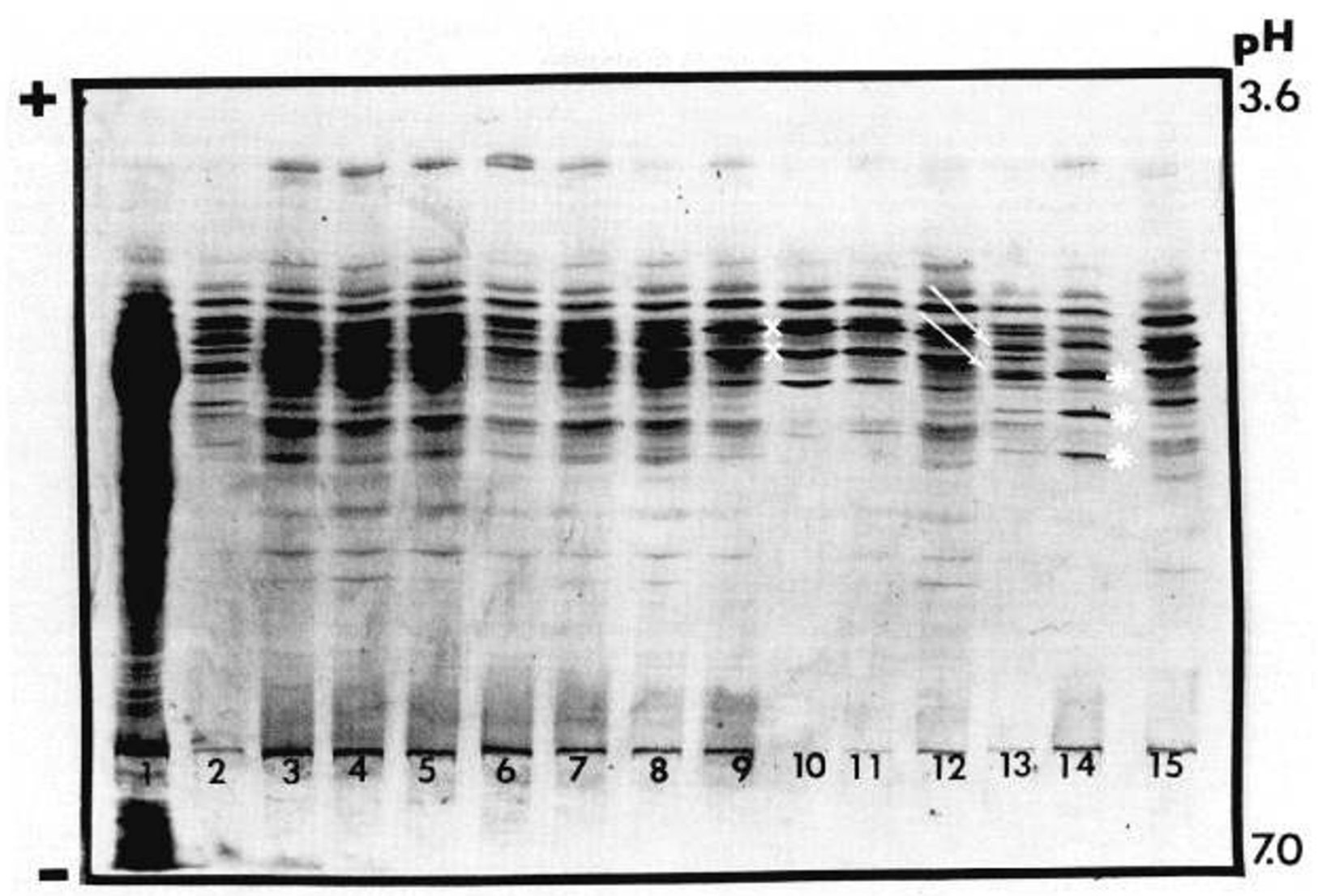

Fig. 2. Differences in the IEF profile of MACRODUCT collected sweat obtained from sexually mature males and females. Sweat samples from five females and nine males. all over $18 \mathrm{yr}$ of age, were collected following pilocarpine stimulation of the forearm utilizing the MACRODUCT collection system. Twenty microliters of these samples were subjected to IEF analysis over a $\mathrm{pH}$ range of 3.6 to 7.0 and the resulting protein patterns visualized by silver staining. The female sweat samples were analyzed in lanes 3 through 7 and displayed consistent profiles. The male sweat samples were analyzed in lane 2 and lanes 8 through 15. The male sweat samples could be divided into three patterns as defined in the results section with major representative differences being noted among each group by the presence of $x$ 's, arrows, or asterisks. Controls included triplicate runs on each sample and triplicate collections to ensure the patterns did not change on a day-to-day basis. Lane 1 represents a pooled normal control serum utilized as a laboratory internal control.

into the blocking solution and gently agitated for $30 \mathrm{~min}$ at room temperature. The membrane was then removed and transferred to a vessel containing the primary antibody and incubated for $30 \mathrm{~min}$ at room temperature. The membrane is then washed three times for $10 \mathrm{~min}$ in Tris buffer containing $0.05 \%$ Tween 20. The membrane was then transferred to the second antibody solution and incubated with gentle agitation for $30 \mathrm{~min}$ at room temperature. The membrane was then washed three times for 10 min at room temperature in Tris buffer containing $0.05 \%$ Tween 20. The membrane was then immersed in HRP Color Development Solution for 15 to $30 \mathrm{~min}$ at room temperature until the desired intensity was obtained. The membrane was then washed with distilled water and allowed to air dry. Controls included incubation with nonimmune rabbit serum in place of immune serum and the "dotting" of buffer solution in place of the sweat sample.

\section{RESULTS}

Electrofocusing of pure and crude sweat. The first goal of this study was to determine if sweat collection methodology would affect the IEF profile of human sweat. Initial experiments performed on both crude and pure sweat samples demonstrated that a $\mathrm{pH}$ gradient of 3.6 to 9.0 allowed the resolution of the majority of the proteins of human sweat (Fig. $1 A-D$ ). No proteins were found which processed isoelectric points below $\mathrm{pH} 3.5$ and above $\mathrm{pH} 9.0$ when a broad $\mathrm{pH}$ range was attempted $\mathrm{pH}$ range 2.5 to 11.0, data not shown). A pH range of 3.5 to 11.0 demonstrated crowding of the bands in the acidic region rendering the resulting IEF profile uninterpretable. Thus, a pH range of 3.6 to 7.0 was employed for the studies reported herein since this gradient gave maximum resolution of the protein bands while including the majority, if not all, of the proteins present in human sweat.

Sweat collected by dripping from the forearm during exercise was compared to that obtained using the MACRODUCT collector following both exercise and pilocarpine stimulation. The results of this study clearly demonstrated that sweat collected from both males and females contained fewer proteins when collected utilizing the MACRODUCT than when collected by dripping from the forearm (Fig. $1 A-D$ ). This increased protein content of sweat collected under crude conditions was especially noticeable in the basic or maybe less acidic pH range of the gel where numerous proteins in low concentration were focused. These proteins were absent in sweat collected utilizing the MACRODUCT. For the crudely collected sweat, there was also a major increase in the protein content of that region of the gel where the majority of the proteins focus. These findings together suggest that sweat collected by dripping from the forearm contains proteins originating from contaminating sites and not from the eccrine sweat gland. Additionally, for MACRODUCT collected sweat, there was little difference in the electrofocusing profile for heat-stimulated versus pilocarpine-stimulated sweat samples, indicating that the method of stimulation has little or no effect on sweat protein composition. The above findings have been confirmed on 24 sweat samples from 11 females and 13 males collected using both the MACRODUCT system and by 
dripping from the forearm. In addition, 10 of the above sweat samples were collected in triplicate on separate days and all triplicates obtained utilizing the MACRODUCT collection system yielded identical IEF profiles. In contrast, similar triplicate samples obtaincd by droplet collection failed to yield consistent IEF profilcs.

Differences in the electrofocusing profle between pure sucat from sevually mature males and females. During the above studies assessing sweat collection methodology, preliminary observations suggested that the IEF pattern was different between male and female sweat collected utilizing the MACRODUCT system. When sweat samples from five females and nine males all over 18 yr of age were focused over the $\mathrm{pH}$ range of 3.6 to 7.0. the difference between male and female protein patterns became obvious (Fig. 2). The female sweat samples demonstrated that, while the amount of total protein in each sample varied, all the samples contained similar protein bands (Fig. 2. lanes 3-7). In contrast, the sweat collected from males demonstrated marked differences in protein pattern among the nine samples collected. $V$ isual analysis of the gel demonstrated that the sweat samples from males could be divided into different patterns. These three patterns are defined by lanes 9 through 12 and 15; lanes 2,8 and 13: and lane 14. The IEF pattern of lanes 9 through 12 and 15 were quite similar and all possessed two major protein bands (Fig. 2, crosses) missing, or in vastly reduced concentration, in all female sweat samples and the remaining male sweat samples. The major difference between the most common male IEF pattern (lanes 9 through 12 and 15) and the next most common pattern (lanes 2,8, and 13) was the presence of additional protein bands not present in the most common profile (Fig. 2, arrows) and a decrease in the major bands noted previously in the most commonly encountered profile (Fig. 2, crosses). The remaining differences between these two patterns are minor and may be due to differences in protein concentration of the sweat samples and not due to the proteins being absent. The least common IEF pattern (lanes 14) possessed little similarity to any other sweat sample whether it was collected from males or females as noted by the increased presence of several protein bands (Fig. 2, asterisks) and the complete lack of several other bands. A close inspection of the medical and ethnic backgrounds of the normal volunteers employed in this study failed to uncover any reason for the differences noted in sweat protein composition. Controls in the above studies included triplicate IEF runs on all the samples to assure that the pattern did not change on a day-today basis, that gel scans agreed with the visual observations, and that the lane position of individual samples had no effect on the IEF profile.

Parameters to judge the purity of human sweat. The methodology supplied by the manufacturer of the MACRODUCT system performs quite well in practice. From our experience, two items are of importance in the collection of sweat free from contaminating macromolecules. These involve thorough washing and rinsing of the skin collection site and firm attachment of the MACRODUCT to the forearm (Fig. 3A). The resulting clear differences in the IEF profiles between sweat samples collected utilizing the MACRODUCT system and simple droplet collection from the skin surface suggested a need for a technique to confirm the purity of collected sweat. Qualities of an ideal
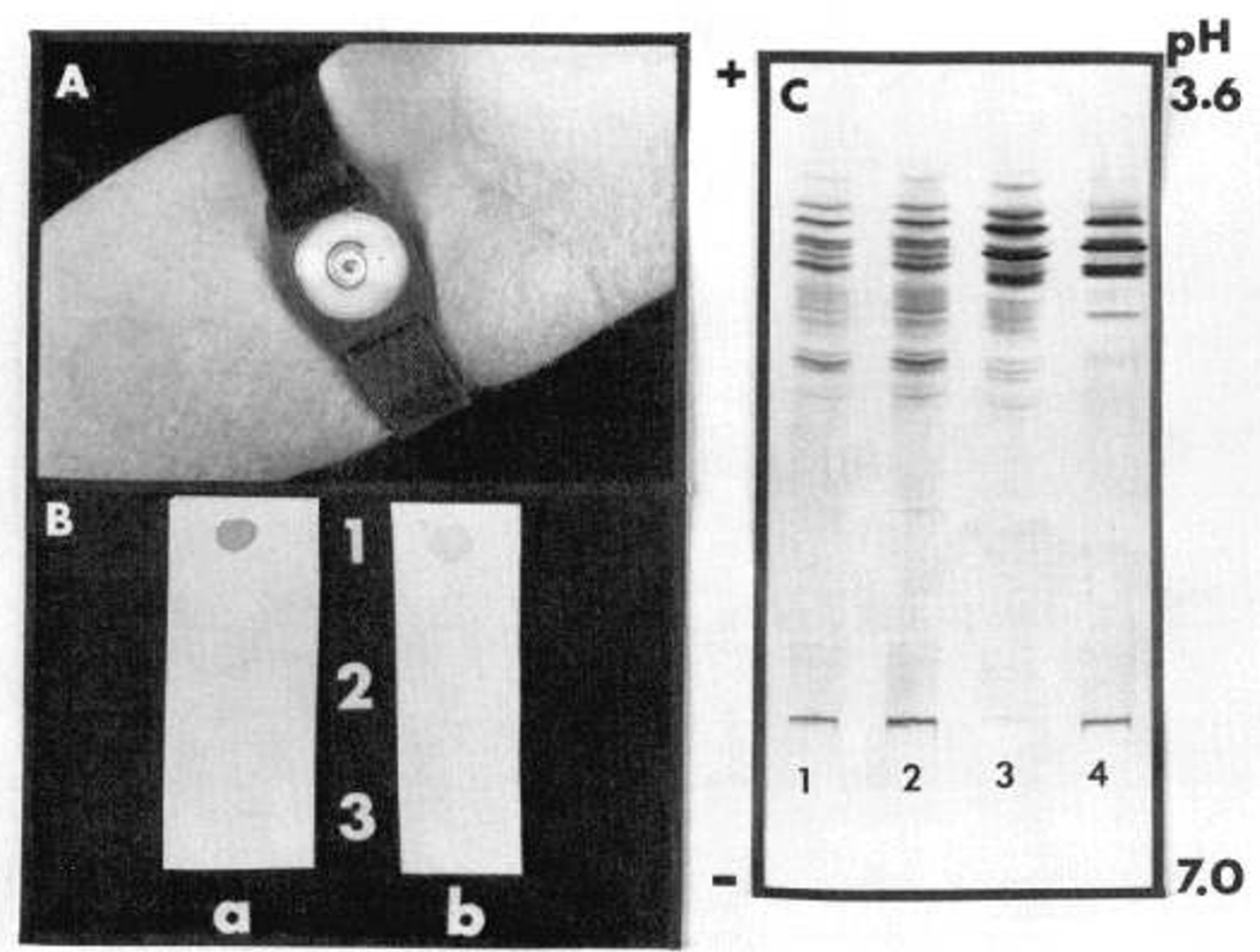

Fig. 3. The placement of the MACRODUCT. parameters to judge sweat purity, and IEF profiles of sexually immature sweat samples. A demonstrates the correct positioning and tightness of the MACRODUCT for collection of sweat from the forcarm area. $B$ - $a$ demonstrates the immuno-dot immunoreactivity for human serum proteins of: l) sweat collected by the droplet method: 2) sweat collected by the MACRODUCT following either exercise: or 3) pilocarpine stimukation. $B$ - $l$ ) demonstrates the immuno-dot immunoreactivity for epidermal keratins of $l$ ) sweat collected by the droplet method: 2) sweat collected by the MACRODUCT following either exercise: or 3) pilocarpine stimulation. ( demonstrates the IFF profile of MACRODUCT collected sweat following pilocarpine stimulation of: $I$ ) a 6 -yt-old female; 2) a 5-yr-old male; 3 ) a 35 -yr-old male; and 4 ) a 15 -yr-old male. $A$ total of 20 prepubertal males and females were analyzed with constant results. 
technique to confirm sweat purity should include the ability to be rapidly performed with commercially available reagents and the ability to perform the test prior to IEF such that valuable technical time is not lost. Since human skin contains an abundance of epidermal keratins, immunostaining for this protein utilizing a dot-immunobinding assay, before IEF analysis, was tested to determine if it would be a parameter to assess the purity of collected sweat. The results depicted in Figure 3B-b clearly demonstrated that sweat collected by droplet collection from the skin surfacc stained heavily for the presence of epidermal keratins. In contrast. sweat collected employing the MACRODUCT system failed to stain, when exposed in an identical fashion, to antisera against epidermal keratins (Fig. $3 B-b$ ). The MACRODUCT collected sweat samples were then concentrated 7-fold and the immuno-dots repeated to access if any keratin could be detected in these concentrated samples. The results of this test demonstrated that a slight staining for keratin could be obtained for MACRODUCT collected sweat, but that this reactivity was well below that obtained from unconcentrated sweat samples obtained by the droplet method (data not shown).

Several other human serum proteins were assessed as potential markers for the purity of sweat samples. These included IgA, albumin, $\alpha-1$-antitrypsin, transferrin, haptoglobin, B2-microglobulin, and $\alpha-1$-acid glycoprotein. While none was present in MACRODUCT collected sweat, all demonstrated a highly variable occurrence in sweat collected by the droplet method, with some samples being highly reactive and others slightly reactive for each antiserum tested. In an effort to overcome the above variability, an identical study was performed utilizing an antiserum to whole human serum in place of antisera to individual serum proteins. As demonstrated in Figure $3 B-a$, only sweat collected by the droplet method was reactive for the presence of human serum proteins. Since slight immunoreactivity was detected on MACRODUCT collected sweat (which could be normal background staining or indicative of a slight reactivity to human serum proteins) the above was repeated on macroduct sweat concentrated 7-fold. The results of this protocol clearly indicated that MACRODUCT collected sweat did react positively, but slightly, for the presence of human serum proteins (data not depicted). In no instance did the amount of reactivity approach that obtained on unconcentrated sweat collected by the droplet method. Thus, an immuno-dot protocol for either the presence of epidermal keratins or whole human serum proteins can scrve as an indicator of sweat purity and, for superior quality control, both can easily be performed.

Differences in the IEF profile between MACRODUCT collected sweat from sexually immature males and females. Studies on 10 prepuberty females demonstrated that the IEF profiles were identical to that found for the mature female sweat samples described previously (Fig. 3c, lane 1 compared to Fig 2, lanes 37). An identical analysis of 10 prepubertal males demonstrated that the two major proteins demonstrated in mature male sweat samples (Fig. 3c. lane 2 compared to lane 3 ) were greatly reduced in concentration in all the samples tested. In addition, the IEF pattern obtained for immature males was identical to that obtained for the entire female sample population tested (Fig. 3c, lane 2). Thus, before puberty, the protein composition of human sweat is identical for the male and female population. Sweat samples were also obtained from 10 males between the ages of 13 and 16 yr who displayed evidence of undergoing puberty and all were demonstrated to have a mature male IEF sweat pattern (Fig. 3c, lane 4). To explore further the differences noted in prepubertal and mature male sweat samples, especially as it applies to the exact time of the protein pattern changes, will require a large expansion of the sample population, a careful assessment of the degree of sexual maturity in young males, and the assessment of the levels of circulating sex hormones in both the adult and juvenile subjects. These studies currently exceed the approved human rescarch guidelines and expanded approval is pending.

\section{DISCUSSION}

The most reliable diagnostic test to confirm the presence of $\mathrm{CF}$ in a particular patient is the documentation of electrolyte abnormalities in eccrine sweat (1). These electrolyte abnormalities have prompted the interest of many research groups to examine sweat obtained from normal and CF patients to determine if differences also exist in the activity, distribution, and composition of the macromolecular components. In the past. this type of analysis has been hindered (due to the extremely low concentration of macromolecular components in sweat) by the lack of a sensitive electrophoretic technique as it applies both to the resolving power and detection of the individual protein bands present in sweat. With the advent of thin-layer IEF and silver diamine staining of polyacrylamide gels the above problems have been largely overcome $(3-5,10,11,13)$. However, the results of the present study clearly demonstrate that no matter how sensitive the physical techniques employed, the attainment of a consistent sample of human sweat is crucial to obtain consistent results in the electrophoretic analysis of the macromolecular components of human sweat.

The extreme importance of the purity of the sweat sample obtained for analysis is best exemplified by the findings in this study of clear differences in the protein composition of male versus female sweat samples. These clear differences between the protein composition of male and female sweat samples are noted only when utilizing the MACRODUCT system for sweat collection. The rationale underlying this finding centers on the fact that the MACRODUCT collection system only collects sweat from a very small area of skin surface $\left(6.20 \mathrm{~cm}^{2}\right)$. This is in direct contrast to the most common technique which collects sweat by droplet collection from the skin surface; a methodology which can cover several hundred square centimeters of surface area. The origin of the contaminating proteins present in sweat collected by the droplet method conceivably arise mostly from the skin surface, hair follicles, and sebaceous glands. Indirect evidence for contamination by skin macromolecules was the finding of abundant keratin immunoreactivity, by immuno-dot assay. in sweat collected by the droplet method, but not in sweat collected by the MACRODUCT system. Contamination by sebaceous secretions and hair follicles of sweat collected by the droplet method was suggested by the milky appearance of the sweat collected, which is characteristic of the secretory product of these glands. In contrast, the sweat collected utilizing the MACRODUCT system was clear and free of particulate matter. Thus, the MACRODUCT sweat collection system appears to allow the convenient collection of human sweat with a high. probability of originating from the human sweat gland.

Once sweat samples were obtained with a high probability of origination from the eccrine sweat gland, several findings of interest were elucidated regarding the sweat protein composition in adults and juvenile subjects. The IEF analysis clearly demonstrated that all female-derived sweat samples had identical protein compositions regardless of age or sexual maturity. The mature male sweat samples, on the other hand, displayed three different protein profiles which were consistent from collection to collection for each individual. The significance of these variant patterns at present is unclear but should resolve as the sample size increases where national origin and genetic backgrounds can be statistically analyzed. These findings again point out the importance of the purity of the sweat sample collected since other reports analyzing the macromolecular components of sweat have failed to note these obvious changes between male and female sweat samples $(3-5,14,15)$.

The finding that sweat samples from males and females differ. and that these differences in males arise upon reaching puberty. suggests that these proteins are under hormonal control mechanisms presenting during sexual development. Depending on the identity of the hormones influencing this altered protein expression several areas of clinical utility might presumably be devel. 
oped. These would include the possible early diagnosis of hormone-producing neoplasms in children and adult women through the inappropriate appearance of an adult male sweat protein pattern; the monitoring of sexual dysfunction to separate metabolic alteration from psychological causes; the possible monitoring of the progression of neuropathy since neurohumoral stimuli to these glands may be blunted; and, the monitoring of nonenzymatic glucosylation of these proteins in diseases such as diabetes mellitus. The relative inexpensive and noninvasive nature of sweat collection methodology renders it ideal for clinical development.

Regarding CF, the results contained herein hopefully will serve to allow the valid collection and interpretation of sweat protein patterns from normal and CF subjects. While a recent study proposes a difference in the protein pattern of sweat obtained from normal and CF individuals in the acidic region of 2dimensional gels, these studies are difficult to interpret due to the collection methodology employed as well as the patient population chosen for study (16). First, sweat was collected by heat stimulation in a sauna bath with collection being attained by droplet collection from the skin surface. Although the authors state that the sensitivity of their electrophoretic technqiue serves as a control for surface contamination by corneocytes or foreign material, no biochemical evidence is presented regarding sweat purity. The authors also state that electrophoretic patterns of whole epidermal cells contain a variety of macromolecules greater in size than albumin and that the absence of these easily detectable sweat proteins indicates a noncontaminated sample of sweat. While possibly true, the authors overlook a possible substantial contamination by sebaceous secretions and hair follicles. In fact, such contamination is suggested by the stated need to centrifuge the sweat samples to remove particles prior to analysis.

In addition, the patient population chosen for study was appropriately based on the analysis of entire families with CF. Unfortunately, no data were presented relating to the patients age, sex, or degree of sexual maturity. It is possible that the differences notes were not due to CF but due to sampling children with $\mathrm{CF}$ who were sexually immature and comparing these profiles to profiles of mature males. In our preliminary studies, it has been demonstrated that even age-matching normal and $\mathrm{CF}$ males is inadequate because of the delay of sexual maturity in $\mathrm{CF}$ patients presumably due to the common problem of malnutrition. Thus, for the collection and analysis of sweat protein composition in disease states, the utilization of the MA-
CRODUCT collection system along with careful attention to sex, age, and sexual maturity should greatly improve the documentation of disease-related differences in sweat protein composition.

Acknowledgments. The authors acknowledge the helpful discussion of Drs. Milton Westphal, Margaret Jenkins, and Phillipe Arnaud. The authors also thank Ms. Sandy Varner for excellent editorial assistance.

\section{REFERENCES}

1. Di Sant'Agnes PA, Darling RC, Perera GA, Shea E 1953 Abnormal electrolyte composition of sweat in cystic fibrosis of the pancreas: clinical significance and relationship to disease. Pediatrics 12:549-563

2. Di Sant'Agnes PA, Talmo RC 1967 Pathogenesis and physiopathology of cystic fibrosis of the pancreas. N Engl $J$ Med 277:1287-1294

3. Rubin RW, Penneys NS 1983 Subpicogram analysis of sweat proteins using two-dimensional polyacrylamide gel electrophoresis. Anal Biochem 131:520-524

4. Marshall T 1984 analysis of human sweat proteins by two-dimensional electrophoresis and ultrasensitive silver staining. Anal Biochem 139:506-509

5. Allen RC, Arnaud P. Spicer SS 1981 Affinity-immunodeletion (AID) isoelectric focusing on ultrathin gels applied to the identification of sweat, saliva and blood proteins using silver diamine staining. Electrophoresis 2:167-180

6. Shulz IJ 1969 Micropuncture studies of the sweat information in cystic fibrosis patients. J Clin Invest 48:1470-1477

7. Mangos JA, McSherry NR 1967 Sodium transport: inhibitory factor in sweat of patients with cystic fibrosis. Science 158:135-136

8. Kaiser D, Drack E, Rossi E 1971 Inhibition of net sodium transport in single sweat glands by sweat with cystic fibrosis of the pancreas. Pediatr Res 5:167172

9. Saul A, Don M 1984 A rapid method of concentrating proteins in small volumes with high recovery using Sephadex G-25. Anal Biochem 138:451453

10. Merril CR, Goldman D, Sedman S, Ebert MH 1981 Ultrasensitive stain for proteins in polyacrylamide gels shows regional variation in cerebrospinal fluid proteins. Science 216:1437-1438

11. Emerson DL, Cellier-Chapuis C, Arnaud P 1980 Horizontal two-dimensional (iso-dalt) electrophoresis: Use of agarose isoelectric focusing and SDS gel electrophoresis in an exponential gradient for characterization of plasma proteins. Electrophoresis 1:159-163

12. Gershoni JM, Palde GE 1983 Review: protein blotting: principles and applications. Anal Biochem 131:1-15

13. Allen RC 1980 Rapid isoelectric focusing of detection of nanogram amounts of proteins from body tissues and fluids. Electrophoresis 1:32-37

14. Sano K. Nakao M, Shiba A, Kobayashi K 1984 An ultramicroassay for proteins in biological fluids other than blood using a combination of agarose gel isoelectric focusing and silver staining. Clin Chim Acta 137:115-122

15. O'Neal C, Page JR, Remington JS 1967 Immunologic studies in normal human sweat. J Lab Clin Med 69:631-650

16. Penneys NS, Haft J, Rubin R 1984 Electrophoretic patterns of proteins in cystic fibrosis sweat. J Invest Dermatol 83:238-239 